\title{
ENERGY-EFFICIENT TASK SCHEDULING ALGORITHMS FOR CLOUD DATA CENTERS
}

\author{
T. Jenifer Nirubah ${ }^{1}$, Rose Rani John ${ }^{2}$ \\ ${ }^{I}$ Post-Graduate Student, Department of Computer Science and Engineering, Karunya University, Tamil Nadu, India \\ ${ }^{2}$ Assistant Professor, Department of Computer Science and Engineering, Karunya University, Tamil Nadu, India
}

\begin{abstract}
Cloud computing is a modern technology which contains a network of systems that form a cloud. Energy conservation is one of the major concern in cloud computing. Large amount of energy is wasted by the computers and other devices and the carbon dioxide gas is released into the atmosphere polluting the environment. Green computing is an emerging technology which focuses on preserving the environment by reducing various kinds of pollutions. Pollutions include excessive emission of greenhouse gas, disposal of e-waste and so on leading to greenhouse effect. So pollution needs to be reduced by lowering the energy usage. By doing this, utilization of resources should not be reduced. With less usage of energy, maximum resource utilization should be possible. For this purpose, many green task scheduling algorithms are used so that the energy consumption can be minimized in servers of cloud data centers. In this paper, ESF-ES algorithm is developed which focuses on minimizing energy consumption by minimizing the number of servers used. The comparison is made with hybrid algorithms and most-efficient-server first scheme.
\end{abstract}

Keywords: Cloud computing, Green computing, Energy-efficiency, Green data centers and Task scheduling.

\section{INTRODUCTION}

Cloud computing is a computing model which is developed based on other computing models like grid computing, distributed computing, parallel computing, cluster computing, etc [1]. The main goal of cloud computing is to use the resources in an efficient way and also to gain large profit [2]. The principle behind cloud computing is pay for what is used. In cloud environment, the users can share data and resources among them. The major services provided by the cloud are Saas (Software as a Service), Iaas (Infrastructure as a Service), Paas (Platform as a Service). The datacenters in Clouds has increased the usage of computers in each year, which increases the consumption of energy and also negative impact on the environment [3]. While speaking about energy consumption, green computing technology must be included as it deals with reducing pollution by conserving energy. Green computing can refer to energy-efficient personal computers. The purposes of energy consumption reduction are: minimizing performance losses, achieving target battery lifetime, satisfying performance requirements, minimizing power consumption, minimizing the $\mathrm{CO}_{2}$ emissions, maximizing the profit, maximizing resource utilization [4]. As the number of users in cloud is increasing, the tasks to be scheduled also increases. The performance of the cloud is dependent on the task scheduling algorithms that is being used [5]. Cloud servers are normally implemented in one or more data centers. The major component of a datacenter in energy consumption is the servers. The usage of servers in an inefficient manner leads to more energy being consumed [6]. In data centers, the reduction in energy consumption can be done by reducing the number of active servers. Initially more servers are provided to meet the increase in demand. So many task scheduling algorithms have been developed and proposed so far with the aim of reducing the energy consumption. All the task scheduling algorithms are developed in such a way that the incoming tasks will be scheduled based on a procedure given by the algorithm. Also the condition that the tasks should meet with the deadline is essential. In [7], many green task scheduling algorithms are developed with continuous and discrete speeds on heterogeneous computers. Such algorithms are needed so that the emission of green house gas being emitted in the atmosphere can be reduced by lowering the energy consumption. Data centers have many servers. Energy consumed by the servers is increasing to a greater extent. A task scheduling algorithm is needed for minimizing energy consumption in cloud computing data centers. With the help of the task scheduling algorithm, the tasks can be scheduled to a minimum number of servers by maintaining the task response time constraints. The number of servers that can be active in order to meet the time constraints must be taken into consideration. Data centers that enhance the green computing technology can be called as Green Data Centers. The main aim is to minimize the allocated number of servers and thus to save the energy. Data center comprises of many computer systems and components for communication, processing and storage. A data center size may vary like occupying one room of a building, one or more floors or an entire building [8]. In order to maintain the energy efficiency in data centers, various factors are needed to be considered. If there are any servers that are not in use, then they can be 
turned off. Some servers are under-utilized and they run at a maximum of 15 percent. Hence tasks in such servers can be given to other high efficient servers which are having less space and so the former servers can also be turned off [9]. The servers which process huge number of tasks will be more efficient in terms of utilization rate. Inefficient power supplies can also result in wasting half of the power before it goes to the server. The tasks given to the servers should be scheduled within the particular processing time. The data center consists of many switches and routers which enables communication between the servers. In data centers, the task scheduling and allocation to servers is done with the help of a scheduling algorithm. Users send their request to the data center for computing whatever task they want to get executed. The task may be reading file contents, updating data, uploading files, downloading software, etc. The data center will classify the tasks based on the scheduling algorithm that is used and then allocates the task to one of the available servers. The servers that are active can be reduced in order to conserve energy. Energy consumption can be maintained by making the active servers to continue their work. ESF-ES algorithm is developed by using the parameters from hybrid algorithms [7] and mostefficient-server-first scheme algorithm [6].

\section{RELATED WORK}

A cloud computing system contains large number of heterogeneous servers which execute tasks that are assigned to them and consumes large amount of power. This is due to the poor task assignment optimization. When the power consumption is high, then the energy emission is high and the carbon dioxide gas also will be emitted more into the environment causing pollution. Therefore it is necessary to develop efficient green computing algorithms to lower the energy consumption on heterogeneous cloud servers. In [10], some of the green task scheduling algorithms is used to minimize the energy consumption in heterogeneous cloud servers. In [11], green task scheduling algorithms with speed adjustment and maximum speeds are used to reduce energy consumption in heterogeneous computers and to finish the tasks before deadline. In [7], several green task scheduling algorithms are used in which some algorithms work for continuous speeds and other algorithms for discrete speeds. All these algorithms focus on minimizing the consumption of energy and reducing the emission of carbon dioxide gas into the atmosphere. A green scheduling algorithm is given in [3] which use a neural network predictor to predict the future load demand and the focus is on energy shortage and global climate change problems. In [6], most-efficient-server first scheme is used where energy consumption of servers in data centers is calculated. The study of efficient task scheduling in order to minimize the data center server energy consumption is focused which can be achieved by reducing the number of servers. In [12], various power-aware algorithms are used with dynamic voltage and speed which are applicable to task scheduling in real-time multiprocessing systems and communication environments. Task scheduling using genetic algorithm is discussed in $[1,13]$ where the concern is about efficient allocation and scheduling of tasks. Slack reclamation is the execution of individual tasks with slacks. This concept is used by $[14,15]$ to focus on the energy consumption problems in parallel and distributed computing systems with DVFS method. The problem of power consumption in high performance processors is addressed in[16] which also uses slack reclamation. In [17], the need for scheduling algorithms in order to minimize the wasted server energy is considered. Energy-conscious scheduling algorithms are used to minimize energy consumption in servers of data centers [18]. Power consumption problem is considered in [19] and is optimized through priority-based preemptive scheduling algorithm. Power-aware scheduling algorithms with deadline constraints for heterogeneous systems are used in [20] to meet the deadline constraints in high performance computing applications. Stochastic Max-Min and Min-Min algorithms are used to solve the problem of scheduling multiple, divisible independent tasks on a heterogeneous distributed computing system [21]. A task clustering method is used in [22] to solve the problem of task assignment in heterogeneous computing systems and the tasks are assigned to the processors in such a way that the communication cost and total execution cost are minimized.

\section{ESF-ES ALGORITHM}

ESF_ES algorithm is developed by combining the hybrid algorithm and most-efficient-server first scheme and is implemented in this paper. Set of tasks and servers are taken as input. The scheduling of tasks to the servers and the data center server energy consumption is given as output of the algorithm. The users will request for computing various types of tasks. Each task may fall under a particular task type like reading file contents, updating data, uploading files, downloading software, etc. Based on the type of task selected, the processing time vary. The number of instruction in each task is obtained. Energy slope is calculated for each task of different types in each server with the help of processing time. Energy consumption is calculated by using the number of instructions and the energy slope. Task allocation is done in such a way that most-efficient-server gets the tasks first. Number of active servers among the set of available servers is reduced. The algorithm follows a greedy approach.

\section{ESF-ES algorithm}

\footnotetext{
Input: Set of tasks and servers.

Output: Scheduling of tasks to servers and data center server energy consumption.

Get the number of tasks, number of available servers, task type of each task and the number of instruction of each task. $\mathrm{a}=\mathrm{b}=1$

for each task $\mathrm{x}$ of type i do

for each server $k$ do
} 
Calculate $\gamma_{i, k}=C_{i}\left[p t_{i, k}\right]^{\alpha_{i}-1}$

Calculate server energy consumption

$$
E(i, k)=R_{i, k} \gamma_{i, k}
$$

if $\mathrm{E}(\mathrm{i}, \mathrm{k})<=\mathrm{E}(\mathrm{a}, \mathrm{b})$ then

$a=i, b=k$

end

end

end

Output the task allocation to the servers and the total energy consumption by the servers.

$p t_{i, k}$ is the processing time for task of type ' $\mathrm{i}$ ' at server ' $\mathrm{k}$ ', $R_{i, k}$ is the number of instructions of each task and $\gamma_{i, k}$ is the energy slope of task ' $\mathrm{i}$ ' in server ' $k$ '. This algorithm is helpful in completing the sequential tasks on the heterogeneous servers in order to minimize the consumption of energy in a cloud computing scenario. Heterogeneous tasks are handled in an efficient way. As like the algorithm in [6], this algorithm also will use only the servers that are necessary. Additional servers are taken only if the current servers are not adequate.

\section{SIMULATION RESULTS AND}

\section{PERFORMANCE ANALYSIS}

The simulation of ESF-ES green task scheduling algorithm for a data center with heterogeneous tasks is done using java and so that the server energy consumption can be reduced to a certain extent. The reason for calling as green task scheduling algorithm is that when the energy consumption is reduced, the carbon dioxide gas emission can also be reduced resulting in the enhancement of green computing technology. An energyefficient task scheduling algorithm namely ESF-ES is implemented in this paper. Input is the number of servers which handles heterogeneous tasks. The number of servers is taken as 5. Four different task types are considered namely reading file contents, updating data, uploading files and downloading software. The algorithm is implemented in Java. The processing time (ms) taken for each task varies depending upon their task types. For every simulation, the processing time (pt) and the number of instructions for each task (R) are randomly generated. The processing time for the task type reading file contents ranges between $1000 \leq \mathrm{pt} \leq 1200$; for updating data, $1300 \leq \mathrm{pt} \leq 1500$; for uploading files, $1600 \leq \mathrm{pt}$ $\leq 1900$; for downloading software, $2000 \leq \mathrm{pt} \leq 2500$. The number of instruction for each task varies $1000 \leq \mathrm{R} \leq 9000$, $1000 \leq C_{i \leq 1500}$ and $3 \leq \alpha_{i} \leq 4$. The comparison is made between three algorithms namely hybrid algorithm, mostefficient-server first scheme and ESF-ES algorithm. The energy consumption of data center servers is measured in joules $(\mathrm{J})$ and the processing time is measured in milli seconds (ms). The processing time is used for calculating the energy slope which in turn used in the calculation for energy consumption.

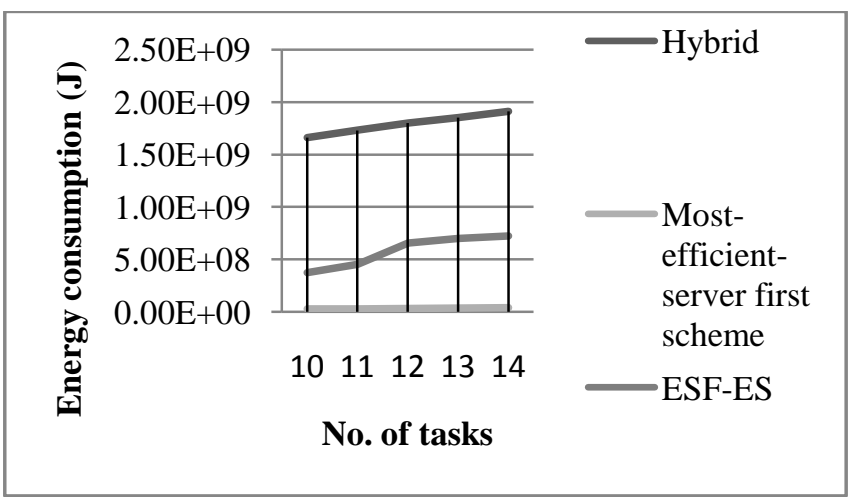

Chart -1: Comparison based on number of tasks

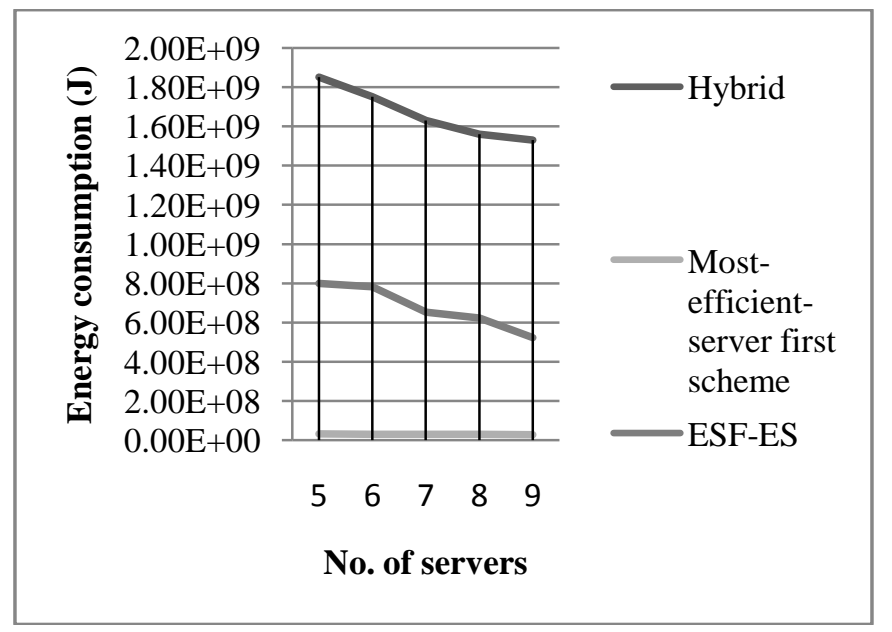

Chart -2: Comparison based on number of servers

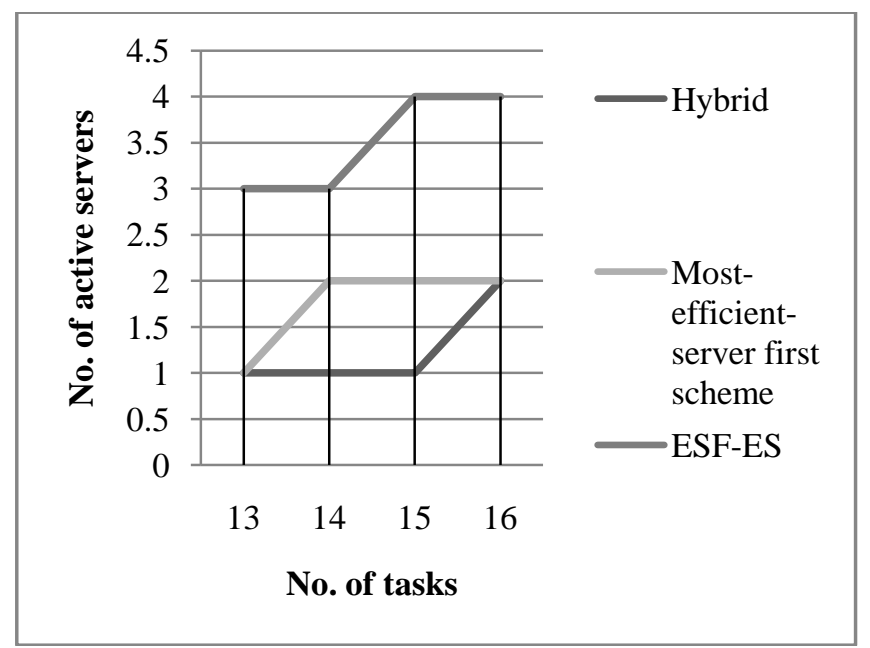

Chart -3: No. of active servers vs. no. of tasks 
In the first two graphs, the server energy consumption is shown where the number of servers is fixed to 5 and the number of tasks is varied from 10 to 14 in the first graph. As the number of tasks to scheduled increases, the energy consumption also increases. The hybrid algorithm is consuming more energy than the other two algorithms. New algorithm is better in energy conservation when compared to hybrid algorithm. But most-efficient-server first scheme still proved to be the best among the three algorithms. In the second graph, the number of tasks is fixed to 12 and the number of servers varies from 5 to 9 . As the number of servers increases, the energy consumption decreases. Again mostefficient-server first scheme is better than the newly developed algorithm and the hybrid algorithm. The third graph shows the number of servers that are used when the number of tasks is changed.

\section{CONCLUSIONS}

The various energy-efficient task scheduling algorithms are studied. The ESF-ES algorithm is implemented which uses a greedy task scheduling scheme and compared with the mostefficient-server first scheme algorithm and the hybrid algorithms. In the hybrid algorithm, only the number of tasks is considered. In the most-efficient-server first scheme and the ESF-ES algorithm, different types of tasks are also included. When the result analysis is made, even though hybrid algorithm is the best among many other green task scheduling algorithms, it consumes more energy when compared to the ESF-ES algorithm and most-efficient-server first scheme. The ESF-ES algorithm consumes more energy than the mostefficient-server first scheme. By comparing all the three algorithms, the result obtained is that the most-efficient-server first scheme algorithm is best in conserving energy in servers of cloud data centers. In this way, task scheduling can be done in an efficient way and the tasks are allocated to servers in such a way that the energy consumption is greatly reduced.

\section{REFERENCES}

[1] Junwei GE, Yongsheng Y., (2013) "Research of cloud computing task scheduling algorithm based on improved genetic algorithm", in: Proceedings of the 2nd International Conference on Computer Science and Electronics Engineering.

[2] Chawla Y. and Bhonsle M., (2012) "A Study on Scheduling Methods in Cloud Computing", International Journal of Emerging Trends \& Technology in Computer Science (IJETTCS) Volume 1, Issue 3, September - October.

[3] Truong V.T.D., Sato Y., Inoguchi Y., (2010) "Performance evaluation of a green scheduling algorithm for energy savings in cloud computing", in: Proc. 2010 IEEE International Symposium on Parallel \& Distributed Processing, Workshops and Phd Forum (IPDPSW), April, pp. 1-8.
[4] Beloglazov A., Buyya R., Lee Y.C., Zomaya A., (2011) "A Taxonomy and Survey of Energy-Efficient Data Centers and Cloud Computing Systems", Advances in Computers, Volume 82, Pages 47-111.

[5] Lepakshi V.A., Dr. Prashanth C S R, (2013) "A Study on Task Scheduling Algorithms in Cloud Computing", International Journal of Engineering and Innovative Technology (IJEIT) Volume 2, Issue 11.

[6] Liu N., Dong Z., Rojas-Cessa, R., (2013) "Task Scheduling and Server Provisioning for EnergyEfficient Cloud-Computing Data Centers," Distributed Computing Systems Workshops (ICDCSW), 2013 IEEE 33rd International Conference on, vol., no., pp.226,231, 8-11.

[7] Zhang L.M., Li K., Lo D.C., Zhang Y., (2013) "Energy-efficient task scheduling algorithms on heterogeneous computers with continuous and discrete speeds", Sustainable Computing: Informatics and Systems 3, 109-118.

[8] http://en.wikipedia.org/wiki/Data_center.

[9] http://searchitchannel.techtarget.com/feature/Upgradin g-to-energy-efficient-servers

[10] Zhang L.M., Li K., Zhang Y.-Q., (2010) "Green task scheduling algorithms with speeds optimization on heterogeneous cloud servers", in: Proc. of 2010 IEEE/ACM International Conference on Green Computing and Communications (Green-Com2010), Hangzhou, December 18-20, pp. 76-80.

[11] Zhang L.M., Li K., Zhang Y.-Q., (2010) "Green task scheduling algorithms with energy reduction on heterogeneous computers", in: Proc. of 2010 International Conference on Progress in Informatics and Computing (PIC-2010), Shanghai, December 1012, pp. 560-563.

[12] Li K., (2008) "Performance analysis of power-aware task scheduling algorithms on multiprocessor computers with dynamic voltage and speed", IEEE Transactions on Parallel and Distributed Systems 19 (11) 1484-1497.

[13] Omara F.A., Arafa M.M., (2010) “Genetic algorithms for task scheduling problem, Journal of Parallel and Distributed Computing", Volume 70, Issue 1, Pages 13-22 http://dx.doi.org/10.1016/j.jpdc.2009.09.009.

[14] Rizvandi N.B., Taheri J., Zomaya A.Y., (2011) "Some observations on optimal frequency selection in DVFSbased energy consumption minimization", Journal of Parallel and Distributed Computing 71 (8) 1154-1164.

[15] Rizvandi N.B., Taheri J., Zomaya A.Y., Lee Y.C., (2010) "Linear combinations of DVFS-enabled processor frequencies to modify the energy-aware scheduling algorithms", in: Proc.10th IEEE/ACM International Conference on Cluster, Cloud and Grid Computing, pp. 388-397.

[16] Zhu D., Melhem R., Childers B.R., (2003) "Scheduling with dynamic voltage/speed adjustment using slack reclamation in multiprocessor real-time systems", IEEE 
Transactions on Parallel and Distributed Systems 14 (7) 686-700.

[17] Wang L., Khan S.U., Chen D., Kolodziej J., Ranjan R., Xu C., Zomaya A., (2013) "Energy-aware parallel task scheduling in a cluster", Future Generation Computer Systems, Volume 29, Issue 7, Pages 1661-1670.

[18] Lee Y.C., Zomaya A.Y., (2009) "On effective slack reclamation in task scheduling for energy reduction", Journal of Information Processing Systems 5 (4) 175186.

[19] Shin Y., Chois K., Sakurait T., (2000) "Power optimization of real-time embedded systems on variable speed processors", in: IEEE/ACM International Conference on Computer Aided Design, pp. 365-368.

[20] Ma Y., Gong B., Sugihara R., Gupta R., (2012) "Energy-efficient deadline scheduling for heterogeneous systems", Journal of Parallel and Distributed Computing, 72, 1725-1740.

[21] Kamthe A., Lee S.-Y., (2007) "Stochastic approach to scheduling multiple divisible tasks on a heterogeneous distributed computing system", in: Proc. IEEE International Parallel and Distributed Processing Symposium, pp. 1-11.

[22] Ucar B., Aykanat C., Kaya K., Ikincib M., (2006) "Task assignment in heterogeneous computing systems, Journal of Parallel and Distributed Computing", Volume 66, Issue 1, Pages 32-46. 